\title{
Editorial
}

\section{Supporting Databases for Neuroscience Research}

The provisional completion of the Human Genome Project in the past year has attested to the power of databases to become essential tools in biological research. Much of this effectiveness has derived from the innate simplicity of the sequence data. However, neuroscience data present much more difficult problems, which thus far have been difficult to solve. Although the emergence of electronic versions of journals is a step in the right direction, the data in an article still remain locked within the text. Simple search tools can identify key words and terms in titles, abstracts, and articles, but structured databases and sophisticated search tools are still lacking on a broad scale that will provide for massive and efficient movement of information out of journals and into the hands of research workers.

The first steps in this direction are being taken by the Human Brain Project and other federally funded initiatives. These have begun to develop pilot databases and informatics tools for different types of neuroscience data that have the potential to facilitate research at different levels of study. One of the most challenging areas is brain imaging. The complex two-dimensional and fourdimensional imaging data contrast sharply with the single dimension of sequence data; in addition, they involve extremely large graphics files, complex problems of comparison between data sets, and many related problems.

The need for appropriate databases to facilitate the analysis of brain images, comparisons between results between different laboratories, development of consensus standards, and reduction of duplication in research efforts has been increasingly recognized. To meet that need, a functional magnetic resonance imaging (fMRI) Data Center (www.fmridc.org) has been established as a publicly accessible repository of fMRI data. According to the organizers, "The fMRI Data Center, funded by the National Science Foundation and Keck Foundation, exists with the goal of providing access to a common data set that other researchers, from a variety of fields, can use to develop and evaluate methods, e.g. new statistical techniques, as well as perform metaanalyses. Such data sets are common in the meteorological, genetics, and high energy physics communities."

The second year of operation of the fMRI Data Center has been reviewed recently by the External Advisory Committee, chaired by Marcus Raichle. (The report, as well as the full membership of the committee, is listed at the website.) The committee gives a strong endorsement to the Center in working toward its goals: "first, to provide a publicly accessible repository of peer-reviewed fMRI studies, and second, to provide all data necessary to interpret, analyze, and replicate these studies." The committee noted strong progress in essential areas, including smoothly functioning data-acquisition procedures, accessibility of the data to users, outreach to encourage the imaging community to share data, establishment of a Summer Workshop on fMRI informatics, development of data-management software with dissemination to individual laboratories, and development of tools that allow researchers to summarize and compare data sets. The committee noted that work still lies ahead in encouraging researchers to share data through databases such as this and in obtaining a consensus on conventions for describing experiments to facilitate analysis and comparisons between laboratories.

In response to a request to comment on whether journals should require authors to deposit fMRI data to the Center in conjunction with publication, similar to the depositing of sequence data in sequence databases, the committee recommended informal encouragement rather than formal guidelines at this stage.

In summary, the advisory committee, representing the imaging community, has given a strong endorsement to the continued development of the fMRI Center.

The success of a Center such as this will be in direct proportion to the amount of data that is submitted to it. The Journal of Neuroscience has been among the leaders in moving into the electronic publishing era. It is therefore appropriate for the Journal to support initiatives such as this to make the data in our flagship journal for the Society for Neuroscience more accessible and more useful in promoting research on the nervous system.

To support this initiative, the Journal of Neuroscience therefore encourages authors of fMRI papers accepted for publication in our journal to submit the experimental data (e.g. images, statistical overlays, and behavioral data) pertaining to their paper to the fMRI Data Center at the above URL. We believe that it will not be necessary to make this a requirement, because the advantages of participation will be immediately obvious. As usual, we solicit feedback from members of the Society for Neuroscience in developing further this policy.

We anticipate that this will be the first of a series of databases for different types of neuroscience data that we will recommend to our authors. Development of these databases will forge ever closer bonds between journals and research workers to enhance the use of the results of our studies to further our insights into brain function.

Gordon M. Shepherd

Editor-in-Chief 